\title{
Excited meson spectroscopy with chirally improved fermions*
}

\section{T. Burch ${ }^{\dagger}$, C. Hagen, D. Hierl, and A. Schäfer}

Institut für Theoretische Physik

Universität Regensburg

D-93040 Regensburg, Germany.

E-mail: tommy.burch@physik.uni-r.de

Christof Gattringer, L. Ya. Glozman

Institut für Physik, FB Theoretische Physik

Karl-Franzens-Universität Graz

A-8010 Graz, Austria.

We present excited meson masses from quenched calculations using chirally improved (CI) quarks at pion masses down to $350 \mathrm{MeV}$. The salient features of our analysis are the use of a matrix of correlators from various source and sink operators and a basis which includes quark sources with different spatial widths, thereby improving overlap with states exhibiting radial excitations.

XXIIIrd International Symposium on Lattice Field Theory

25-30 July 2005

Trinity College, Dublin, Ireland

\footnotetext{
*For the Bern-Graz-Regensburg (BGR) Collaboration.

${ }^{\dagger}$ Speaker.

${ }^{\ddagger}$ Supported by Fonds zur Förderung der Wissenschaftlichen Forschung in Österreich, project P16823-N08.
} 


\section{Introduction}

The spectroscopy of excited hadrons still poses a significant problem to the lattice QCD community. Since a Euclidean-time hadron correlator is composed of a sum of contributions from the different mass eigenstates, each of which being exponentially suppressed by a factor of the mass, disentangling the higher mass states from those of the dominant ground state (let alone the other excited masses) can be a daunting task.

In the following, we present a method (see also [1]) for handling just this "disentanglement" of masses and we show our latest results for the meson sector. In another contribution to this conference [2], we present the results for baryons.

\section{Method}

Our method is based upon the variational approach developed by Michael [3] and later refined by Lüscher and Wolff [ [ correlators which we then diagonalize.

The basic structure of our meson interpolators is

$$
O=\bar{u}_{n, w} \Gamma d_{n, w},
$$

where we use $\Gamma=\gamma_{5}$ and $\gamma_{4} \gamma_{5}, \gamma_{i}$ and $\gamma_{4} \gamma_{i}, \gamma_{5} \gamma_{i}, \gamma_{i} \gamma_{j}$, and 1 for pseudoscalar (PS), vector (V), pseudovector (PV), tensor (T), and scalar (SC) mesons, respectively. The subscripts $n$ and $w$ refer to narrow and wide quark sources. These are created using gauge-covariant Jacobi smearing to approximate Gaussian distributions of widths 0.27 and $0.41 \mathrm{fm}$. By opening this new degree of freedom to the mesons, we hope to improve overlap with states exhibiting radial excitations (a difference in sign between the narrow and wide source contributions will create a radial node in the corresponding quark wavefunction). We use degenerate $u$ and $d$ quark masses. For strange mesons, we replace one of the light quarks by the strange quark (the physical strange quark mass is determined via the PS kaon).

For our non-strange PS and V mesons we then have up to 6 different interpolators ( $n w=w n$ for degenerate quark masses) for our basis, giving up to a $6 \times 6$ correlator matrix (this becomes $8 \times 8$ for the strange mesons). For the PV, T, and SC mesons, we have a basis of up to 3 interpolators (4 for the strange mesons).

After creating our correlator matrix, $C(t)$, we must then solve the generalized eigenvalue problem:

$$
C(t) \vec{v}^{(k)}=\lambda^{(k)}(t) C\left(t_{0}\right) \vec{v}^{(k)},
$$

where the $\lambda^{(k)}(t)$ are the eigenvalues corresponding to the eigenvectors $\vec{v}^{(k)}$. We note in passing that we arrive at almost identical eigenvalues, up to a constant factor, with a normal eigenvalue problem, i.e., by omitting the matrix $C\left(t_{0}\right)$ on the r.h.s. (the eigenvectors, however, are a different matter). The eigenvalues provide the time dependence, and hence the mass, of the separate mass eigenstates:

$$
\lambda^{(k)}(t) \propto e^{-t M_{k}}\left[1+\mathscr{O}\left(e^{-t \Delta M_{k}}\right)\right] .
$$

The eigenvectors provide the corresponding wavefunctions (within the highly limited basis). The $\mathscr{O}\left(e^{-t \Delta M_{k}}\right)$ terms in Eq. (2.3) can be reduced by changing, even by further limiting, the starting 


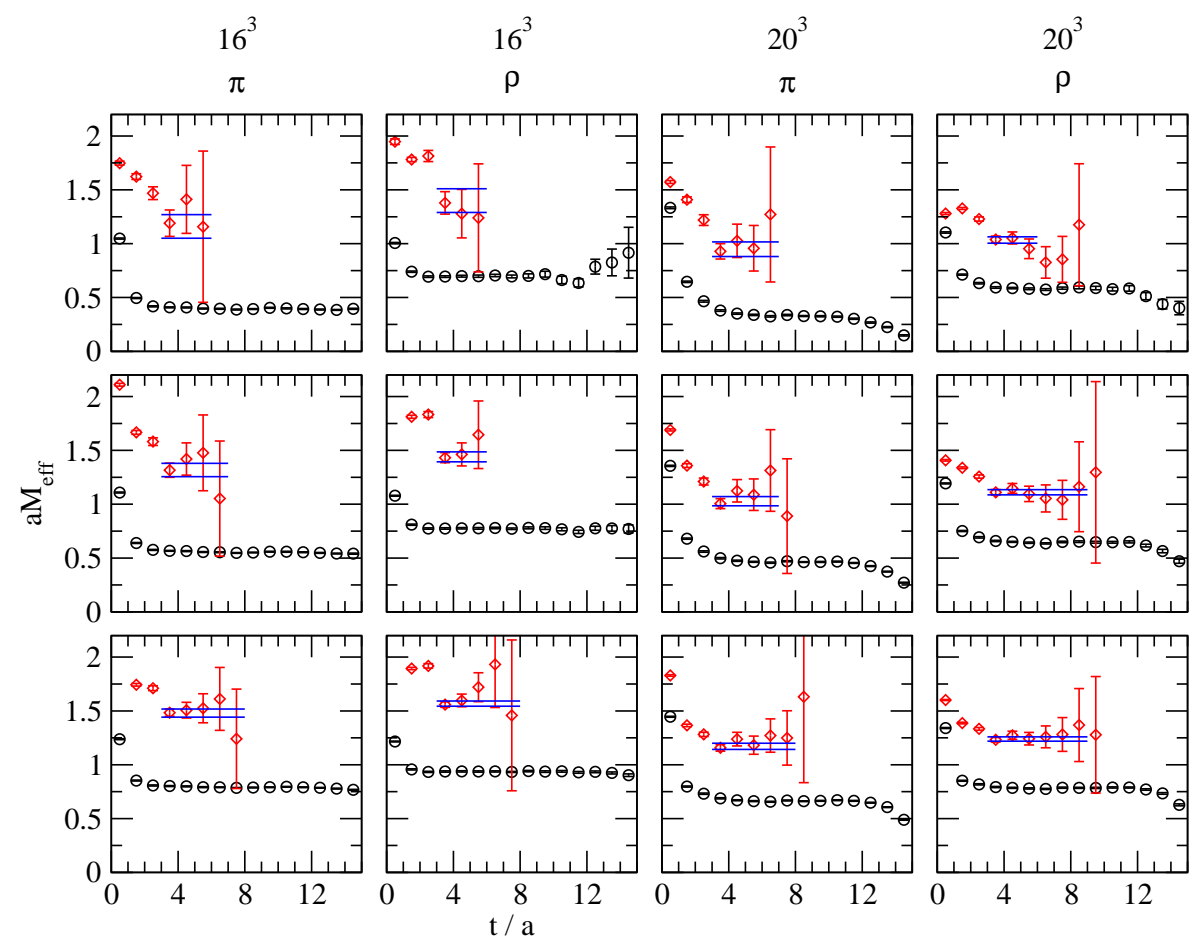

Figure 1: Effective mass plots for pseudoscalar and vector mesons from our coarse $\left(16^{3} \times 32\right.$, with $a m_{q}=$ $0.05,0.1,0.2$ from top to bottom $)$ and fine $\left(20^{3} \times 32\right.$, with $\left.a m_{q}=0.04,0.08,0.16\right)$ lattices. Both ground and excited states are shown, along with the $M \pm \sigma_{M}$ results (horizontal lines) from fits of the eigenvalues in the corresponding time intervals.

basis. We observe such effects in our results and, in the end, see the best effective mass plateaus in the excited-state eigenvalues by choosing $3 \times 3$ correlator matrices throughout $(n \Gamma n, n \Gamma w, w \Gamma w$; the only exceptions are for the PS and V mesons on our coarse lattice, where we use 4 basis states: $\left.n \Gamma n, n \gamma_{4} \Gamma n, n \gamma_{4} \Gamma w, w \gamma_{4} \Gamma w\right)$.

We have results from two sets of quenched configurations, created using the Lüscher-Weisz gauge action. The lattice spacings are determined via the Sommer parameter [5]: $a=0.148 \mathrm{fm}$ for the $16^{3} \times 32$ and $a=0.119 \mathrm{fm}$ for the $20^{3} \times 32$ (both spatial volumes are $\approx 2.4 \mathrm{fm}$ ). The quark propagators are created using the chirally improved (CI) action [6].

\section{Results}

In Fig. 1, we show some of our effective mass plots for the PS and V mesons. Results are shown for both the first and second eigenvalues, corresponding to the ground and first-excited states. From the apparent plateaus, we choose appropriate time intervals over which we fit the eigenvalues with an exponential and extract the mass. 


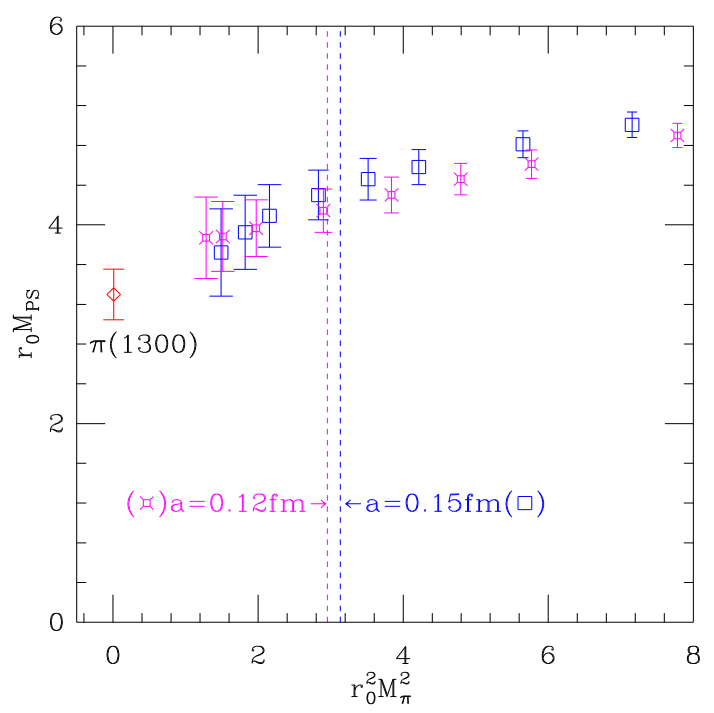

Figure 2: Excited-state pseudoscalar masses vs $M_{\pi}^{2}$ for both lattice spacings. The quark masses are degenerate. All quantities are in units of the Sommer parameter $r_{0}$. The diamond represents the experimental point and the vertical lines mark the values of $r_{0}^{2} M_{\pi}^{2}$ corresponding to the physical strange quark mass.

Repeating this excercise for many quark masses, we arrive at plots like that of Fig. 2, where the excited-state PS mass is displayed as a function of the ground-state PS mass squared $\left(\propto m_{q}\right)$. Although the statistical errors are substantial, the masses appear to be extrapolating to a value consistent with the physical one. Also, the results from the two lattice spacings appear to be consistent with each other, suggesting small discretization effects for this state.

In Fig. 3, similar plots are shown for the V, PV, T, and SC states. For the V mesons, the results are roughly consistent with the experimental values, although it is apparent that discretization effects are larger for these excited states. The other states are more problematic. The excited PV is obviously higher than one might naively expect and results for the other excited states are virtually non-existent (few reliable effective mass plateaus could be found). We plan to improve the picture for such states by including more "realistic" interpolators (e.g., p-wave sources). For the SC meson at small quark masses, ghost states dominate the correlation function (which then becomes negative [7]), and we therefore plot only the results at large quark masses.

Figure $\emptyset$ shows the same plots for the strange PS and V mesons. Again, the excited PS results are consistent with the experimental value and display small discretization effects. The excited V mesons, however, appear to remain high and also suffer more from discretization effects.

When looking at the eigenvectors in the $3 \times 3$ smearing basis alone, invariably we find the same sign for all components in the ground state but sign differences for the excited state. In other words, within this limited basis, the excited state has a radial node in its wavefunction. Including different spin structures $\left(\Gamma\right.$ and $\left.\gamma_{4} \Gamma\right)$ in the PS or the $\mathrm{V}$ basis, the interpretation becomes more ambiguous.

Of course, all of these results are from quenched lattices with relatively small volumes $(\approx 2.4$ $\mathrm{fm})$. So we have some systematic effects which are rather difficult to quantify without repeating our analysis on larger, dynamical configurations. It is quite possible that the small volume may be 

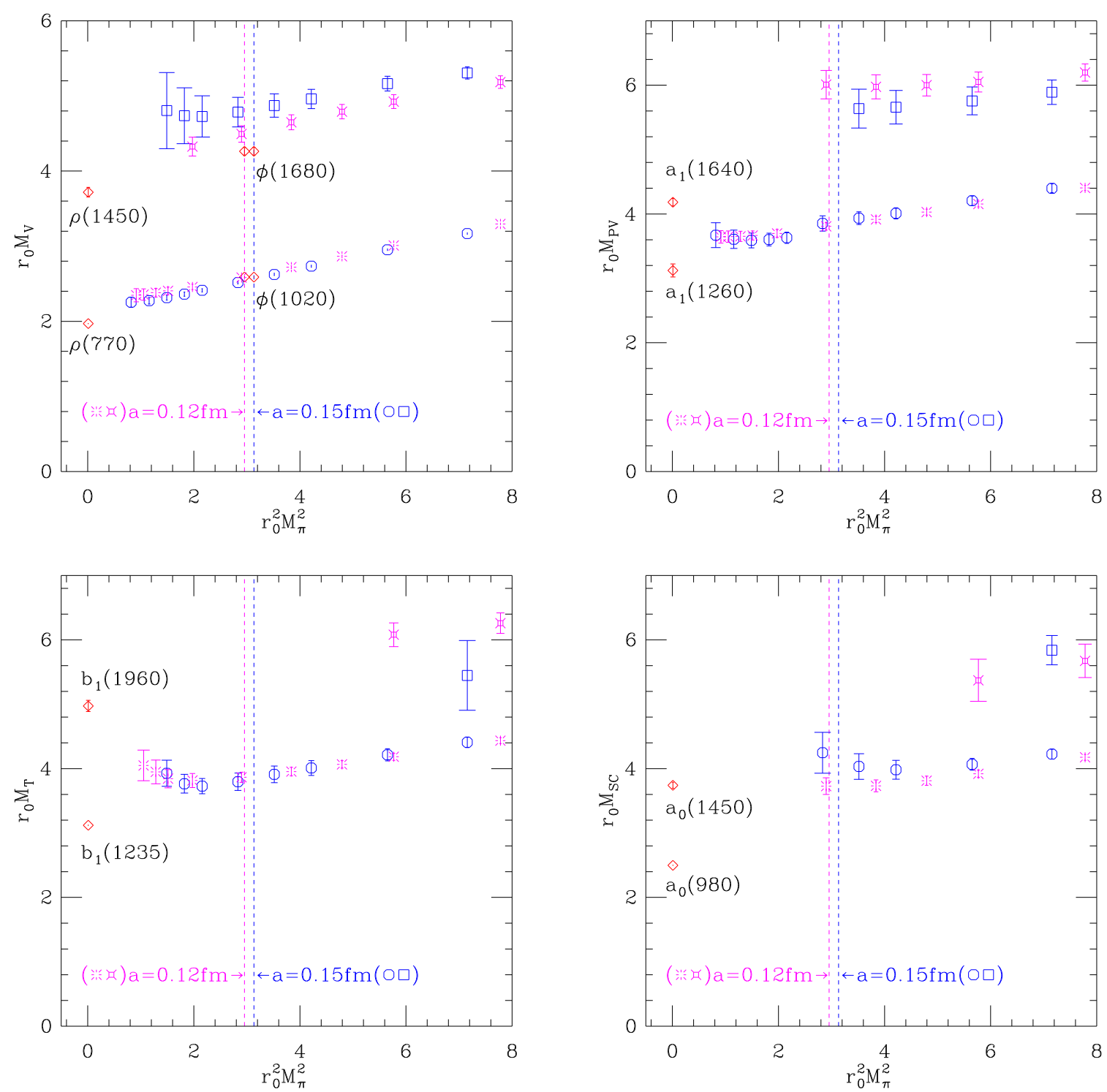

Figure 3: Ground- and excited-state meson masses vs $M_{\pi}^{2}$ for both lattice spacings. Vector, pseudovector, tensor, and scalar mesons appear. The quark masses are degenerate. All quantities are in units of the Sommer parameter $r_{0}$. The diamonds represent the experimental points and the vertical lines mark the values of $r_{0}^{2} M_{\pi}^{2}$ corresponding to the physical strange quark mass.

the reason the excited $\mathrm{V}$ and PV mesons remain higher than the experimental values.

\section{References}

[1] T. Burch, et al. [BGR (Bern-Graz-Regensburg) Collaboration], Spatially improved operators for excited hadrons on the lattice, Phys. Rev. D 70 (2004) 054502 [hep-lat/ 04050 06]; Excited hadrons from improved interpolating fields, Nucl. Phys. Proc. Suppl. 140 (2005) 284 [hep-lat/ 0409014 ]; Masses of excited baryons from chirally improved quenched lattice QCD, Nucl. Phys. A 755 (2005) 481 [nucl-th/ 0501025 ]. 

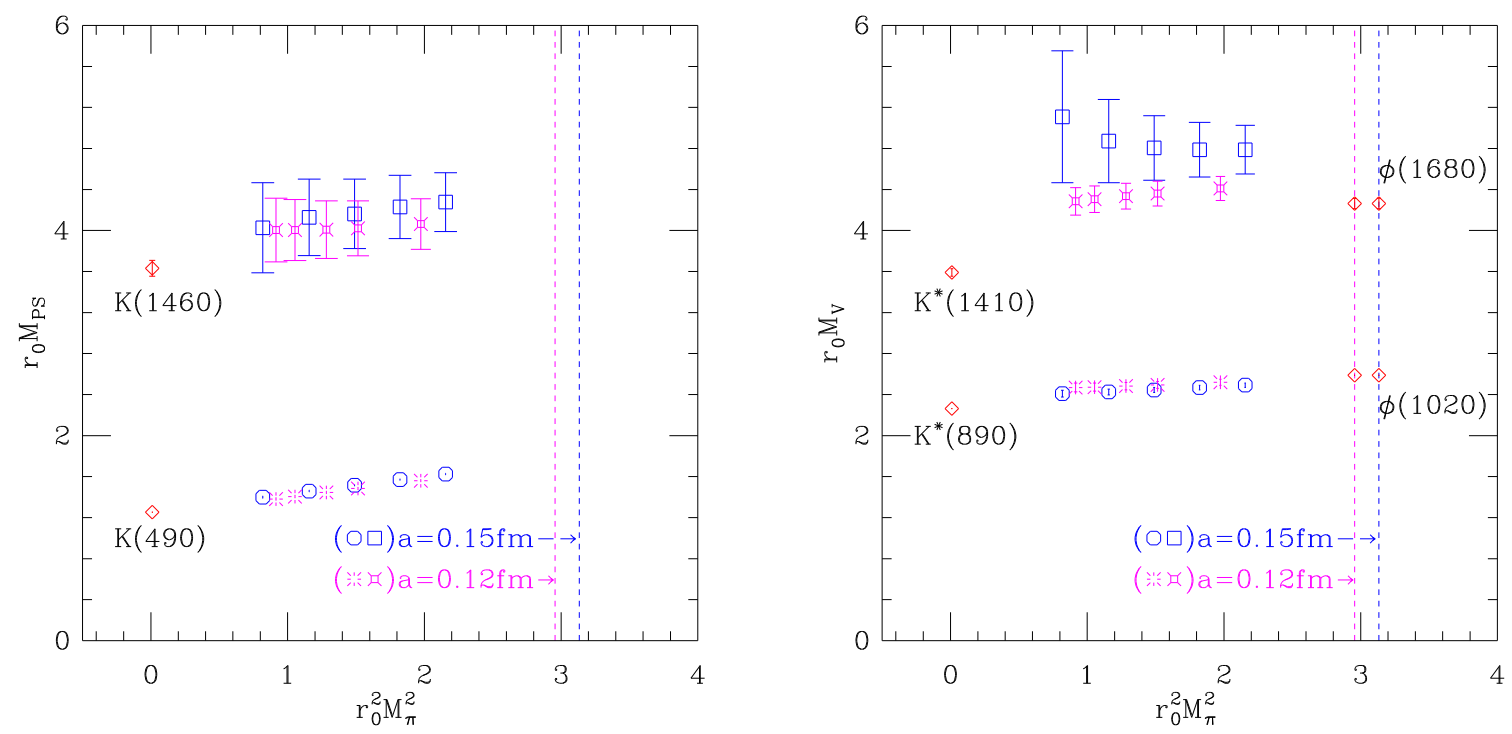

Figure 4: Ground- and excited-state meson masses vs $M_{\pi}^{2}$ for both lattice spacings. Both pseudoscalar and vector mesons are shown. One quark mass is fixed to the physical strange quark mass. All quantities are in units of the Sommer parameter $r_{0}$. The diamonds represent the experimental points and the vertical lines mark the values of $r_{0}^{2} M_{\pi}^{2}$ corresponding to the physical strange quark mass.

[2] T. Burch, et al. [BGR (Bern-Graz-Regensburg) Collaboration], Baryon spectroscopy with spatially improved quark sources, PoS(LAT2005)075.

[3] C. Michael, Adjoint sources in lattice gauge theory, Nucl. Phys. B 259 (1985) 58.

[4] M. Lüscher and U. Wolff, How to calculate the elastic scattering matrix in two-dimensional quantum field theories by numerical simulation, Nucl. Phys. B 339 (1990) 222.

[5] C. Gattringer, R. Hoffmann, and S. Schaefer, Setting the scale for the Lüscher-Weisz action, Phys. Rev. D 65 (2002) 094503 [hep-lat/ 0112024 ].

[6] C. Gattringer, A new approach to Ginsparg-Wilson fermions, Phys. Rev. D 63 (2001) 114501 [hep-lat/ 0003005$]$; C. Gattringer, I. Hip, and C. B. Lang, Approximate Ginsparg-Wilson fermions: A first test, Nucl. Phys. B 597 (2001) 451 [hep-lat / 0007042 ].

[7] W. A. Bardeen, A. Duncan, E. Eichten, N. Isgur and H. Thacker, Chiral loops and ghost states in the quenched scalar propagator, Phys. Rev. D 65 (2002) 014509 [hep-lat/ 0106008 ]. 